\title{
HIPERIDROSE PRIMÁRIA E QUALIDADE DE VIDA
}

Rafael de Oliveira Pena Neto, Simone Shirasaki Orosco

Universidade do Oeste Paulista - UNOESTE. Curso de Medicina, Presidente Prudente - SP. E-mail: rafaelpneto@hotmail.com

\begin{abstract}
RESUMO
A hiperidrose é caracterizada pela transpiração em excesso, de forma incontrolável, podendo surgir sem qualquer fator desencadeante. Atualmente, a simpatectomia é padrão-ouro no tratamento definitivo dos casos mais graves. Diante disso, este trabalho tem como objetivo identificar a frequência de ocorrência de hiperidrose compensatória em pacientes pós simpatectomia e o grau de satisfação do paciente após procedimento. Para o alcance do objetivo, a metodologia escolhida foi a revisão integrativa. Foram selecionados 10 artigos no período de 2007 a 2014. Todos os estudos indicaram que a hiperidrose compensatória é o principal problema em pacientes que são submetidos à simpatectomia, com valores entre $37 \%$ a $100 \%$. A maior parte dos pacientes submetidos à simpatectomia, independente da hiperidrose reflexa, notaram melhora em suas vidas. Devido a grande incidência e impacto na vida social dos portadores, devem-se buscar novas pesquisas para o desenvolvimento de técnicas e tratamentos que diminuam os efeitos da hiperidrose.
\end{abstract}

Palavras-chave: Hiperidrose, Simpatectomia, Sudorese, Qualidade de vida, Satisfação do paciente.

\section{HYPERHIDROSIS AND QUALITY OF LIFE}

\begin{abstract}
Hyperhidrosis is characterized by excessive sweating, uncontrollably, which may arise without any triggers. Currently, sympathectomy is the gold standard for definitive treatment of the most severe cases. Thus, this study aims to identify the frequency of occurrence of compensatory hyperhidrosis after sympathectomy and the degree of satisfaction after the procedure. To reach the objective, the methodology chosen was the integrative review. 10 articles were selected from the period 2007 to 2014 . All studies indicated that compensatory sweating is the worst problem in patients who undergo sympathectomy, with values between $37 \%$ to $100 \%$. Most patients who were undergone to sympathectomy, independent of reflex hyperhidrosis, noticed improvement in their lives. Due to the high incidence and impact on the social life, new researches must be done to develop new techniques and treatments to decrease the effects of hyperhidrosis.
\end{abstract}

Keywords: Hyperhidrosis, Sympathectomy, Sweating, Quality of life, Patient's satisfaction. 


\section{INTRODUÇÃO}

A hiperidrose é caracterizada pela transpiração (sudorese) em excesso, de forma incontrolável, podendo surgir sem qualquer fator desencadeante.

Possui incidência superior a $1 \%$ da população ${ }^{1,2}$.

Segundo Fenili et al. há uma prevalência pouco maior nos indivíduos do sexo masculino em relação ao sexo feminino $^{3}$. Já para Stori et al. a incidência da hiperidrose é pareada entre ambos os sexos, porém o problema é mais evidenciado nas mulheres, pois elas procuram mais auxílio médico devido a um maior constrangimento sentido com esta alteração ${ }^{4}$.

De acordo com uma pesquisa desenvolvida por Hornberger et al., os efeitos maléficos da hiperidrose são comparáveis com os de condições como psoríase severa, insuficiência renal em estágio final, artrite reumatoide e esclerose múltipla ${ }^{5}$.

A hiperidrose pode ser classificada em primária ou secundária. A hiperidrose primária é de maior relevância clínica. Não possui origem conhecida, mas está ligada à hiperatividade do sistema nervoso simpático ${ }^{6}$.

Apresenta-se geralmente localizada, simétrica, e pode afetar inúmeras partes do corpo como: axilas, palmas das mãos, plantas dos pés, face e dorso. Fatores genéticos também podem estar intrinsecamente ligados à hiperidrose primária.

O tratamento clínico pode ser tópico, elétrico ou sistêmico. Em casos moderados algumas opções tópicas podem ser suficientes. A iontoforese é um método simples, com ação apenas em casos leve a moderados. O uso de medicação sistêmica se limita as altas taxas de efeitos colaterais. A toxina botulínica tipo A é uma opção que apresenta inúmeras vantagens, porém é de alto custo e efeito temporário ${ }^{7}$.

Ainda não foi encontrado um tratamento clínico satisfatório para essa condição, por isso, faz-se necessário procedimento cirúrgico para correção do quadro. Atualmente, a simpatectomia é padrão-ouro no tratamento definitivo dos casos mais graves de hiperidrose, principalmente, palmar e axilar, atingindo um grau de efetividade acima de $98 \%{ }^{8}$.

A principal complicação da simpatectomia, sem dúvida, é a sudorese reflexa ou compensatória, sendo a mais comum e presente em grande parte dos pacientes que foram submetidos aos procedimentos de simpatectomia ${ }^{9}$. Os sinais e sintomas aparecem logo após a cirurgia, podendo piorar com alterações climáticas e emocionais, sendo mais comum na porção inferior do tórax, região dorsal, abdome, região lombar, cintura pélvica, fossa poplítea e membros inferiores ${ }^{10}$. 
Diante disso, surge a seguinte questão: qual a frequência de ocorrência de hiperidrose compensatória em pacientes pós simpatectomia torácica e/ou lombar e qual o grau de satisfação do paciente após o procedimento?

Como visto, a hiperidrose é uma doença frequente na população, assim como a busca por tratamentos para resolução dela. Mesmo com o risco de hiperidrose compensatória pós-cirurgia o grau de satisfação parece ser elevado.

Assim, esse estudo tem como objetivo identificar a frequência de ocorrência de hiperidrose compensatória em pacientes pós simpatectomia torácica e/ou lombar e qual o grau de satisfação do paciente após o procedimento.

\section{METODOLOGIA}

Para o alcance do objetivo, a metodologia escolhida foi a revisão integrativa. Esta surgiu devido a crescente quantidade e complexidade de novas informações na área da saúde ${ }^{11,12}$.

A organização do trabalho seguiu seis fases: elaboração da pergunta norteadora, busca ou amostragem na literatura, coleta de dados, análise crítica dos estudos incluídos, discussão dos resultados e apresentação da revisão integrativa ${ }^{12}$.

Como ponto guia da revisão integrativa, formularam-se as seguintes questões: qual a frequência de ocorrência de hiperidrose compensatória em pacientes pós simpatectomia torácica e/ou lombar e qual o grau de satisfação do paciente após o procedimento?

O levantamento bibliográfico foi feito através de bases de pesquisa de artigos científicos. Utilizou-se BVS (Biblioteca Virtual em Saúde) e SciELO (Scientific Electronic Library Online). As palavras-chave utilizadas foram: "Hiperidrose", "Simpatectomia" e "Qualidade de vida".

Os artigos foram inclusos, para a presente revisão integrativa, segundo os seguintes critérios: artigos publicados em português e inglês; textos completos disponíveis nas bases de dados acima citadas; publicados no período compreendido entre 2007 e 2014; artigos com nível 1 de evidência, ou seja, revisões sistemáticas de múltiplos ensaios clínicos randomizados controlados; artigos que retratassem a temática referente ao objetivo da revisão integrativa. Os critérios de exclusão foram: artigos publicados antes de 2007; artigos que não possuíssem nível 1 de evidência; artigos que divergiam da proposta do estudo.

Para análise e síntese desses estudos foi usado um quadro construído para esse fim, que pôde contemplar alguns aspectos considerados pertinentes: nome dos autores; objetivo; tamanho da amostra; resultados; recomendações/conclusões ${ }^{13}$. 
A análise dos estudos selecionados para esta revisão integrativa foi realizada de forma descritiva, possibilitando reunir o conhecimento produzido por estes artigos e dessa forma, atingir o objetivo que esse modelo propõe.

\section{RESULTADOS}

Foram identificados 1156 artigos na Revisão Integrativa, 19 na Scientific Eletronic Library Online (SciELO) e 1137 na Biblioteca
Virtual em Saúde (BVS). Após a seleção por títulos, resumos disponíveis e ano de publicação (período de 2007 a 2014), permaneceram 18 artigos. Deste grupo foram excluídos 8 por não possuírem relação com o tema. Foram para leitura integral 10 artigos. Os artigos selecionados serão apresentados na Tabela 1, que mostra as variáveis envolvidas nos artigos localizados.

Tabela 1. Apresentação da síntese de artigos incluídos na revisão integrativa

\begin{tabular}{|c|c|c|c|c|}
\hline $\begin{array}{l}\text { Nome dos } \\
\text { autores }\end{array}$ & Objetivo & $\begin{array}{c}\text { Tamanho } \\
\text { da } \\
\text { amostra }\end{array}$ & Resultados & $\begin{array}{l}\text { Recomendações/ } \\
\text { Conclusões }\end{array}$ \\
\hline Yazbek, ${ }^{14}$ & $\begin{array}{l}\text { Comparar duas técnicas cirúrgicas } \\
\text { (níveis de desnervação) } \\
\text { de simpatectomia por videotoracos } \\
\text { copia para tratamento de } \\
\text { hiperidrose palmar ou palmo- } \\
\text { plantar a curto e médio prazo. }\end{array}$ & 60 & $\begin{array}{l}59 \\
\text { pacientes apresenta } \\
\text { ra resolução da } \\
\text { hiperidrose palmar. } \\
\text { Uma falha terapêuti } \\
\text { ca ocorreu no grupo } \\
\text { T3. A maioria dos } \\
\text { pacientes } \\
\text { apresentou } \\
\text { melhora da } \\
\text { hiperidrose plantar } \\
\text { sem diferença entre } \\
\text { os grupos. Após } 20 \\
\text { meses, todos os } \\
\text { pacientes de ambos } \\
\text { os grupos } \\
\text { apresentavam } \\
\text { algum grau de } \\
\text { hiperidrose } \\
\text { compensatória, mas } \\
\text { com menor } \\
\text { intensidade no } \\
\text { grupo T3 } \\
\text { (p=0,0007). A } \\
\text { hiperidrose } \\
\text { compensatória } \\
\text { desenvolveu-se na } \\
\text { maioria dos } \\
\text { pacientes no } \\
\text { primeiro mês do } \\
\text { pós-operatório, } \\
\text { com incidência e } \\
\text { intensidade }\end{array}$ & $\begin{array}{l}\text { Ambas as técnicas são } \\
\text { efetivas para tratar a } \\
\text { hiperidrose palmar. A } \\
\text { complicação mais frequente } \\
\text { foi a hiperidrose } \\
\text { compensatória. } \\
\text { A simpatectomia no nível T3 } \\
\text { apresentou menor } \\
\text { intensidade de HC, mas, } \\
\text { apesar disso, a melhora da } \\
\text { qualidade de vida foi similar } \\
\text { em ambos os grupos. }\end{array}$ \\
\hline
\end{tabular}




\begin{tabular}{|c|c|c|c|c|}
\hline & & & $\begin{array}{l}\text { estáveis com o } \\
\text { passar do tempo. } \\
\text { Verificou-se } \\
\text { melhora da } \\
\text { qualidade de vida } \\
\text { desde a primeira } \\
\text { avaliação de pós- } \\
\text { operatório. }\end{array}$ & \\
\hline $\begin{array}{l}\text { Boscardim, } \\
\text { PCB } \\
\text { Oliveira RA } \\
\text { Oliveira } \\
\text { AAFR Souza } \\
\text { JM } \\
\text { Carvalho, } \\
\text { RG }^{15}\end{array}$ & $\begin{array}{l}\text { Descrever os resultados clínicos e o } \\
\text { grau de satisfação de pacientes } \\
\text { submetidos } \\
\text { à simpatectomia torácica ao nível } \\
\text { de 4ạ e 5a costelas (R4-R5) para o } \\
\text { tratamento da hiperidrose axilar. }\end{array}$ & 118 & $\begin{array}{l}\text { Dos } 118 \text { pacientes } \\
\text { do estudo, } 99 \\
(83,9 \%) \text { e } 81 \\
(68,6 \%) \\
\text { apresentaram } \\
\text { resolução total dos } \\
\text { sintomas no pós- } \\
\text { operatório precoce } \\
\text { e tardio, } \\
\text { respectivamente. } \\
\text { Houve efeito } \\
\text { compensatório em } \\
49 \text { pacientes } \\
\text { (41,5\%) no pós- } \\
\text { operatório precoce } \\
\text { e em } 77 \text { (65,2\%) no } \\
\text { pós-operatório } \\
\text { tardio. Desses } 77, \\
55 \text { ( } 71,4 \%) \\
\text { consideraram esses } \\
\text { efeitos como leves. } \\
\text { No pós-operatório } \\
\text { precoce, } 110 \\
\text { pacientes ( } 93,2 \%) \\
\text { estavam satisfeitos } \\
\text { com os resultados } \\
\text { da cirurgia, } \\
\text { enquanto } 104 \\
\text { pacientes ( } 88,1 \%) \\
\text { mantinham-se } \\
\text { satisfeitos no pós- } \\
\text { operatório tardio. }\end{array}$ & $\begin{array}{l}\text { A simpactetomia ao nível R4- } \\
\text { R5 é eficaz na resolução da } \\
\text { hiperidrose axilar primária. O } \\
\text { grau de satisfação dos } \\
\text { pacientes com os resultados } \\
\text { em longo prazo é alto. O } \\
\text { efeito compensatório leve é } \\
\text { o principal efeito colateral } \\
\text { relacionado a essa técnica. }\end{array}$ \\
\hline $\begin{array}{l}\text { Araújo CAA } \\
\text { Azevedo IM } \\
\text { Ferreira AF } \\
\text { Ferreira } \\
\text { HPC } \\
\text { Dantas } \\
\text { JLCM } \\
\text { Medeiros } \\
\text { AC }^{16}\end{array}$ & $\begin{array}{l}\text { Investigar fatores preditivos para a } \\
\text { hiperidrose compensatória após } \\
\text { a simpaticotomia torácica videoen } \\
\text { doscópica. }\end{array}$ & 80 & $\begin{array}{l}\text { De acordo com os } \\
\text { dados obtidos, } 68 \\
\text { pacientes ( } 85 \%) \\
\text { apresentaram } \\
\text { hiperidrose } \\
\text { compensatória, que } \\
\text { foi classificada } \\
\text { como suave } \\
\text { (33,85\%), } \\
\text { moderada (33,8\%) e } \\
\text { grave (32,4\%). } \\
\text { Quanto aos } \\
\text { resultados da } \\
\text { cirurgia, na } \\
\text { avaliação dos } \\
\text { pacientes, } 70 \text { deles } \\
\text { ( } 87,5 \%) \text { se } \\
\text { consideram } \\
\text { satisfeitos, }\end{array}$ & $\begin{array}{l}\text { Embora a hiperidrose } \\
\text { compensatória seja um efeito } \\
\text { adverso frequente após } \\
\text { a simpatectomia, o grau de } \\
\text { satisfação dos pacientes foi } \\
\text { elevado. Os melhores } \\
\text { candidatos } \\
\text { para simpatectomia toracosc } \\
\text { ópica são mulheres adultas } \\
\text { jovens com IMC } \leq 24,9 \\
\text { kg/m2. }\end{array}$ \\
\hline
\end{tabular}




\begin{tabular}{|c|c|c|c|c|}
\hline & & & $\begin{array}{l}\text { enquanto } 10 \\
\text { pacientes (12,5\%) } \\
\text { disseram estar } \\
\text { insatisfeitos. O grau } \\
\text { de satisfação variou } \\
\text { de acordo com o } \\
\text { sexo, a idade, o IMC } \\
\text { e a extensão da } \\
\text { operação. A } \\
\text { hiperidrose } \\
\text { compensatória foi a } \\
\text { mais intensa no } \\
\text { abdome e dorso, } \\
\text { comparando-se } \\
\text { com as pernas. }\end{array}$ & \\
\hline $\begin{array}{l}\text { Nicoleit AR } \\
\text { Psendziuk C } \\
\text { Galvani GC } \\
\text { Pereira SW } \\
\text { Kestering D } \\
\mathrm{M}^{17}\end{array}$ & $\begin{array}{l}\text { Realizar um estudo investigativo } \\
\text { que possa descrever os fatores } \\
\text { associados à } \\
\text { cirurgia videosimpatectomia toráci } \\
\text { a, em paciente submetidos ao } \\
\text { procedimento para tratamento da } \\
\text { hiperidrose primária. }\end{array}$ & 60 & $\begin{array}{l}\text { Sudorese } \\
\text { compensatória ocor } \\
\text { reu em } 86,7 \% \text { dos } \\
\text { pacientes. Mãos } \\
\text { extremamente } \\
\text { secas, em 31,7\% } \\
\text { dos } \\
\text { pacientes. Houve re } \\
\text { corrência dos } \\
\text { sintomas em } 16,7 \% . \\
\text { Apenas } 2 \text { pacientes } \\
\text { demonstraram } \\
\text { arrependimento } \\
\text { por terem realizado } \\
\text { o procedimento. Os } \\
\text { tipos de hiperidrose } \\
\text { com acometimento } \\
\text { palmar } \\
\text { apresentaram } 8 \\
\text { vezes mais } \\
\text { recorrência, } \\
\text { comparados aos } \\
\text { tipos axilar puro e } \\
\text { craniofacial (RP=8,4 } \\
\text { 1; IC95\%= 1,33- } \\
62,67 ; \text { p=0,009). } \\
\text { Quando se } \\
\text { seccionou T2 e T3, } \\
\text { em média ocorreu } \\
\text { um grau de } \\
\text { sudorese } \\
\text { compensatória } \\
\text { maior, comparado } \\
\text { aos níveis de secção } \\
\text { mais baixos (média } \\
=1,73 ; \text { pKruskal- } \\
\text { Wallis=0,016). Os } \\
\text { níveis de secção } \\
\text { mais altos (quando } \\
\text { incluíam T2 e T3) } \\
\text { apresentaram } 25 \% \\
\text { menos satisfação } \\
\text { completa, } \\
\text { comparados aos }\end{array}$ & $\begin{array}{l}\text { Simpatectomia torácica é um } \\
\text { método seguro e efetivo para } \\
\text { tratamento da hiperidrose. } \\
\text { Apesar das altas taxas de } \\
\text { satisfação geral, a ocorrência } \\
\text { de sudorese compensatória é } \\
\text { alta, então os pacientes } \\
\text { devem ser informados sobre } \\
\text { o potencial para este efeito } \\
\text { colateral. }\end{array}$ \\
\hline
\end{tabular}




\begin{tabular}{|c|c|c|c|c|}
\hline & & & $\begin{array}{l}\text { níveis mais baixos } \\
\text { (RP=0,75; } \\
\text { IC95\%=0,58- } \\
0,97 ; \text { pFisher=0,06). }\end{array}$ & \\
\hline $\begin{array}{l}\text { Montessi J } \\
\text { Almeida EP } \\
\text { Vieira JP } \\
\text { Abreu MM } \\
\text { Souza RLP } \\
\text { Montessi O } \\
\text { VD }^{18}\end{array}$ & $\begin{array}{l}\text { Comparar grau de satisfação dos } \\
\text { pacientes simpatectomizados e } \\
\text { presença de sudorese reflexa, de } \\
\text { acordo com diferentes níveis de } \\
\text { ablação. }\end{array}$ & 521 & $\begin{array}{l}\text { Pós-operatório } \\
\text { ótimo no controle } \\
\text { da hiperidrose } \\
\text { palmar/axilar } \\
\text { em, respectivament } \\
\text { e, } 94 / 82 \% \text { dos } \\
\text { pacientes do grupo } \\
\text { I, 89/89\% do grupo } \\
\text { II e } 80 / 80 \% \text { do } \\
\text { grupo III. Sudorese } \\
\text { reflexa em } 67 \% \\
\text { dos pacientes dos } \\
\text { grupos I e II, caindo } \\
\text { para } 61,29 \% \text { no } \\
\text { grupo III devido à } \\
\text { maior termoablaçã } \\
\text { o a partir de T4. } \\
\text { Ocorreu sudorese } \\
\text { reflexa severa em } \\
\text { 32\% dos pacientes } \\
\text { do grupo I, } 9 \% \text { do } \\
\text { grupo II e } 4 \% \text { do } \\
\text { grupo III. }\end{array}$ & $\begin{array}{l}\text { A simpatectomia propiciou } \\
\text { excelente grau de satisfação } \\
\text { e baixo índice de } \\
\text { complicações. Não houve } \\
\text { diferença na incidência de } \\
\text { sudorese reflexa com } \\
\text { diferentes } \\
\text { níveis seccionados; porém, a } \\
\text { intensidade desta } \\
\text { complicação se mostrou } \\
\text { menor quando optamos por } \\
\text { níveis de bloqueio mais } \\
\text { baixos, principalmente T4. }\end{array}$ \\
\hline $\begin{array}{l}\text { Cardoso PO } \\
\text { Rodrigues } \\
\mathrm{KCL} \\
\text { Mendes KM } \\
\text { Petroianu A } \\
\text { Resende M } \\
\text { Alberti LR }{ }^{19}\end{array}$ & $\begin{array}{l}\text { Avaliar a qualidade de vida e a } \\
\text { presença de compensação pós- } \\
\text { operatória de pacientes } \\
\text { submetidos } \\
\text { a smpatectomia torácica. }\end{array}$ & 50 & $\begin{array}{l}\text { Entre todos os } \\
\text { entrevistados, } 27 \\
\text { (54\%) relataram } \\
\text { que o incômodo } \\
\text { pessoal com o suor } \\
\text { excessivo, os levou } \\
\text { a procurar } \\
\text { atendimento } \\
\text { médico; } 23 \text { (46\%) } \\
\text { tiveram prejuízo na } \\
\text { relação social; } 22 \\
\text { (44\%) tiveram } \\
\text { dificuldade relativa } \\
\text { ao ambiente } \\
\text { escolar, enquanto } \\
20 \text { (40\%) relataram } \\
\text { que o incômodo } \\
\text { causado pela } \\
\text { hiperidrose nas } \\
\text { atividades } \\
\text { relacionadas ao } \\
\text { trabalho levou-o a } \\
\text { procurar } \\
\text { atendimento } \\
\text { médico. } 21 \text { (42\%) } \\
\text { foram considerados } \\
\text { tímidos pelas } \\
\text { pessoas de seu } \\
\text { convívio. } 33 \text { ( } 66 \% \text { ) } \\
\text { começaram a } \\
\text { manifestar a } \\
\text { doença na infância }\end{array}$ & $\begin{array}{l}\text { O tratamento cirúrgico da } \\
\text { hiperidrose melhora a } \\
\text { qualidade de vida, porém a } \\
\text { hiperidrose compensatória } \\
\text { ocorre em quase todos os } \\
\text { pacientes, }\end{array}$ \\
\hline
\end{tabular}




\begin{tabular}{|c|c|c|c|c|}
\hline & & & $\begin{array}{l}\text { (até os } 12 \text { anos); } 15 \\
\text { (30\%) na } \\
\text { adolescência e } \\
\text { apenas um ( } 2 \% \text { ) na } \\
\text { fase adulta. O calor } \\
\text { foi responsável pela } \\
\text { crise de suor em } 17 \\
\text { (34\%) pacientes e a } \\
\text { ansiedade esteve } \\
\text { presente em } 19 \\
\text { (38\%). Irritação } \\
\text { gerava crise de suor } \\
\text { em } 31 \text { (62\%). } \\
\text { Hiperidrose } \\
\text { compensatória } \\
\text { ocorreu em } 39 \\
\text { (78\%) pacientes e } \\
46 \text { (92\%) } \\
\text { consideram que a } \\
\text { qualidade de vida } \\
\text { após a operação } \\
\text { "melhorou". }\end{array}$ & \\
\hline $\begin{array}{l}\text { Fiorelli RKA } \\
\text { Elliot LG } \\
\text { Alvarenga R } \\
\text { MP } \\
\text { Morard MR } \\
\text { S } \\
\text { Almeida CR } \\
\text { Fiorelli SKA } \\
\text { Agoglia } \mathrm{BG}^{2} \\
0\end{array}$ & $\begin{array}{l}\text { Avaliar a eficácia e as mudanças na } \\
\text { qualidade de vida após a cirurgia, } \\
\text { através do uso de } \\
\text { dois questionários, que propõem } \\
\text { mensurá-las diretamente. }\end{array}$ & 70 & $\begin{array}{l}\text { A maioria dos } \\
\text { pacientes era do } \\
\text { sexo feminino } \\
\text { (70\%), apresentava } \\
\text { hiperidrose palmo- } \\
\text { plantar (47,1\%), } \\
\text { com início dos } \\
\text { sintomas durante a } \\
\text { infância ( } 82,9 \% \text { ), } \\
\text { história familiar } \\
\text { positiva (65,7\%), e } \\
\text { alegavam } \\
\text { limitações } \\
\text { moderadas a } \\
\text { intensas nos } \\
\text { âmbitos } \\
\text { social/afetivo } \\
\text { (64,7\%) e } \\
\text { profissional } \\
\text { (70,6\%). A idade } \\
\text { média foi de } 25,4 \\
\text { anos (13-47). O } \\
\text { grau de desconforto } \\
\text { geral promovido } \\
\text { pela hiperidrose } \\
\text { (escala subjetiva de } \\
0 \text { a } 10 \text { ) obteve } \\
\text { média de } 9,4, \text { com } \\
\text { queda } \\
\text { estatisticamente } \\
\text { significativa em } \\
\text { relação ao grau de } \\
\text { desconforto da } \\
\text { sudorese residual } \\
\text { (n=15/média } \\
2,2 / p=0,001 \text { ) e da } \\
\text { sudorese }\end{array}$ & $\begin{array}{l}\text { Os resultados foram } \\
\text { comparáveis aos da literatura } \\
\text { no tocante à eficácia do } \\
\text { procedimento e as suas } \\
\text { complicações, observando-se } \\
\text { importante melhora (91\%) da } \\
\text { qualidade de vida dos } \\
\text { pacientes submetidos ao } \\
\text { tratamento cirúrgico. }\end{array}$ \\
\hline
\end{tabular}




\begin{tabular}{|c|c|c|c|c|}
\hline & & & $\begin{array}{l}\text { compensatória ( } n- \\
50 / \text { média } \\
3,6 / p<0,001 \text { ). Foi } \\
\text { obtido um índice } \\
\text { geral de } 91 \% \\
\text { de melhora na } \\
\text { qualidade de vida. }\end{array}$ & \\
\hline Lima $A G^{21}$ & $\begin{array}{l}\text { Avaliação: da variabilidade sazonal } \\
\text { sobre o grau de resoluções da } \\
\text { sudorese palmar e plantar, bem } \\
\text { como a incidência e a intensidade } \\
\text { do suor reflexo e análise da } \\
\text { influência desses fatores sobre a } \\
\text { satisfação; da dor como fator } \\
\text { relacionado à satisfação imediata; } \\
\text { da segurança dos métodos de } \\
\text { expansão pulmonar pós-operatória } \\
\text { com e sem drenagem pleural e } \\
\text { relação dos mesmos à dor. }\end{array}$ & 75 & $\begin{array}{l}\text { Foram incluídos } 82 \\
\text { pacientes } \\
\text { consecutivos, sendo } \\
\text { que } 80 \\
\text { completaram o } \\
\text { seguimento } \\
\text { imediato e } 75 \\
\text { completaram todo } \\
\text { o seguimento. Os } \\
\text { resultados } \\
\text { operatórios } \\
\text { (resolução palmar e } \\
\text { plantar, presença e } \\
\text { intensidade do suor } \\
\text { compensatório) } \\
\text { tiveram variação } \\
\text { entre todas as } \\
\text { estações do ano. A } \\
\text { satisfação teve igual } \\
\text { variação sazonal e } \\
\text { os fatores que } \\
\text { estiveram } \\
\text { relacionados à } \\
\text { queda da satisfação } \\
\text { foram distintos, } \\
\text { conforme a estação } \\
\text { do ano. A drenagem } \\
\text { pleural tubular } \\
\text { fechada e a } \\
\text { expansão } \\
\text { com Valsalva foram } \\
\text { igualmente } \\
\text { eficientes quanto à } \\
\text { presença de } \\
\text { pneumotórax } \\
\text { residual, porém a } \\
\text { drenagem pleural } \\
\text { em selo d'água } \\
\text { aumentou o tempo } \\
\text { cirúrgico e a dor } \\
\text { pós-operatória nas } \\
\text { primeiras } 12 \text { horas; } \\
\text { residual não } \\
\text { aumentou a dor. }\end{array}$ & $\begin{array}{l}\text { Os melhores resultados } \\
\text { obtidos foram obtidos } \\
\text { imediatamente e na } \\
\text { primavera, os piores no } \\
\text { verão e em nível } \\
\text { intermediário no } \\
\text { outono/inverno. Na } \\
\text { primavera o único parâmetro } \\
\text { relacionado à queda na } \\
\text { satisfação foi a não resolução } \\
\text { plantar; já no verão e } \\
\text { outono/inverno, foi a } \\
\text { presença de suor reflexo, } \\
\text { independente de sua } \\
\text { intensidade. A drenagem } \\
\text { pleural tubular fechada teve } \\
\text { desempenho inferior à } \\
\text { expansão pulmonar sob visão } \\
\text { direta com manobra } \\
\text { de Valsalva. O pneumotórax } \\
\text { residual, não influenciou a } \\
\text { intensidade dor pós- } \\
\text { operatória. }\end{array}$ \\
\hline $\begin{array}{l}\text { Peres MFP } \\
\text { Brandão } \\
\text { BFS } \\
\text { Kauffman P } \\
\text { Campos } \\
\text { JRM } \\
\text { Jatene FB }\end{array}$ & $\begin{array}{l}\text { Avaliar os resultados } \\
\text { das características das cefaleias em } \\
\text { pacientes com hiperidrose antes e } \\
\text { após a simpatectomia. }\end{array}$ & 53 & $\begin{array}{l}\text { Avaliamos os } \\
\text { resultados das } \\
\text { características das } \\
\text { cefaleias em } \\
\text { pacientes com } \\
\text { hiperidrose antes e } \\
\text { após a }\end{array}$ & $\begin{array}{l}\text { A frequência de crises de } \\
\text { cefaleia foi significativamente } \\
\text { reduzida após a cirurgia. A } \\
\text { hiperidrose pode ser uma } \\
\text { comorbidade da enxaqueca, } \\
\text { que compartilham uma } \\
\text { disfunção autonômica. }\end{array}$ \\
\hline
\end{tabular}




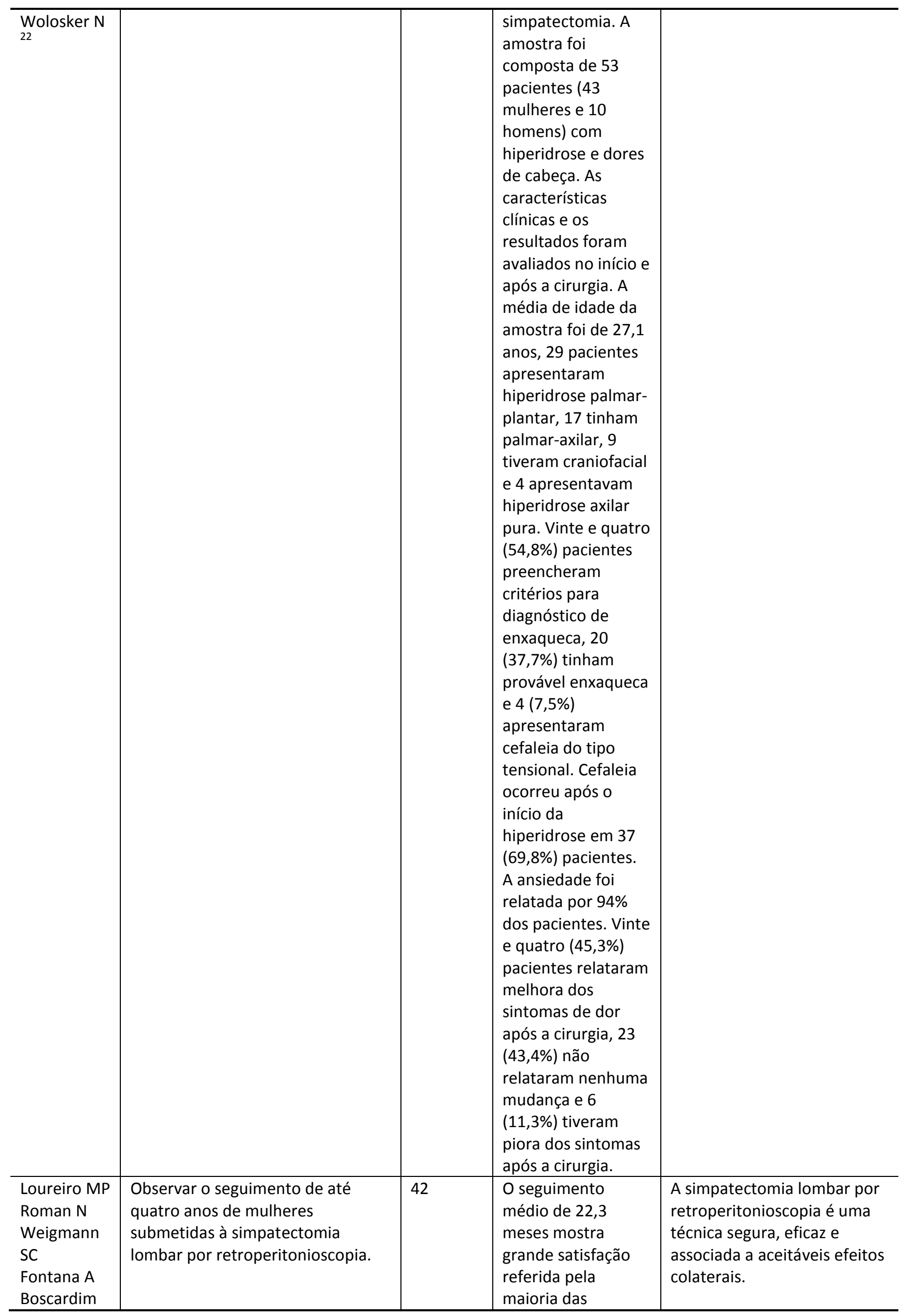




\begin{tabular}{|c|c|}
\hline $\mathrm{PCF}^{23}$ & $\begin{array}{l}\text { pacientes e piora do } \\
\text { suor compensatório } \\
\text { em } 37 \% \text { delas. Não } \\
\text { houve relato de } \\
\text { alteração sexual } \\
\text { nesta casuística. }\end{array}$ \\
\hline
\end{tabular}

Dos artigos incluídos, seis estudos

foram desenvolvidos na região sudeste (60\%), três na região sul (30\%) e um na região nordeste (10\%).

Quanto às publicações, sete aparecem em revistas e arquivos médicos, se destacando o Jornal Brasileiro de Pneumologia; duas são teses de doutorado apresentado à Universidade de São Paulo; e uma em uma revista multidisciplinar.

Em relação ao tipo de delineamento de pesquisa dos artigos avaliados, mostrouse que todos os artigos se enquadram no nível 1 de evidência, ou seja, revisões sistemáticas de múltiplos ensaios clínicos randomizados controlados, com amostra entre 42 e 521 pessoas. Sete trabalhos aceitam como significativo $p<5 \%$, os demais não apresentavam o nível de significância. Os testes estatísticos mais utilizados foram Qui quadrado, teste exato de Fischer e teste T.

Os trabalhos apresentam a porcentagem de homens e mulheres participantes, além da média de idade destes, porém, apenas um inclui a raça dos pacientes submetidos ao procedimento cirúrgico. As mulheres integram a maior parte da amostra de todos os estudos.
Os estudos incluídos na revisão integrativa apresentam revisão bibliográfica, conseguem responder à questão norteadora de seus estudos e apresentam claramente seus resultados, através de gráficos e tabelas. Cinco artigos, além de especificarem o procedimento cirúrgico ao qual o paciente foi submetido, também focam na qualidade de vida e no impacto gerado por esta cirurgia, dessa forma, mostra se o balanço entre os riscos e benefícios foi positivo.

\section{DISCUSSÃO}

A amostra total dos estudos somou 1129 pacientes. Todos estes foram submetidos a algum tipo de simpatectomia para tratamento da hiperidrose primária. Porém, devido ao fato de muitos não especificarem como foram escolhidos esses pacientes, não se pode afirmar que essa amostra foi representativa da população que é portadora de hiperidrose.

A videosimpatectomia torácica e lombar apresentou-se como uma técnica segura $^{17,18,23}$. No estudo de Yazbek foram feitos duas incisões no tórax do paciente, onde por um introduzia-se uma câmera de vídeo e pelo outro um bisturi elétrico ${ }^{14}$. 
Todos os estudos indicaram que a hiperidrose compensatória é o principal problema em pacientes que são submetidos à simpatectomia para tratamento de hiperidrose primária, com valores variando entre $37 \%$ a $100 \%$ (média de $69,17 \%$ ). Essa porcentagem varia de acordo com a técnica utilizada, a altura da secção dos gânglios autonômicos simpáticos e a sazonalidade ${ }^{14,15,18,21}$.

Os estudos mostraram que a secção dos nervos simpáticos na altura de T3 e T4 mostram menor taxa de hiperidrose compensatória ou menor intensidade desta ${ }^{14,18}$. Os piores resultados no nível de hiperidrose compensatória foram obtidos quando a cirurgia foi feita no verão ${ }^{21}$.

Já o grau de satisfação foi avaliado através de questionários não específicos e específicos 20 em cinco estudos. A porcentagem de satisfação variou entre $87,5 \%$ e $97 \%$ (média de $91,12 \%$ ). Isso mostra que a maior parte dos pacientes submetidos à simpatectomia, independente da hiperidrose reflexa, notaram melhora em suas vidas. Um estudo, concluiu que os pacientes submetidos à simpatectomia, além de terem melhoras nos sintomas da hiperidrose, também notaram melhoras nos sintomas de enxaqueca ${ }^{22}$. Assim, a qualidade de vida dos pacientes se elevou de um modo significativo após o procedimento cirúrgico.

\section{CONCLUSÃO}

Concluindo a presente revisão integrativa nota-se que as publicações brasileiras nessa área ainda não possuem grande representatividade. Devido a grande incidência na população e seu impacto na vida social dos portadores, devem-se buscar novas pesquisas para o desenvolvimento de técnicas e tratamentos que diminuam os efeitos da hiperidrose compensatória e aumente ainda mais o grau de satisfação dos pacientes submetidos ao procedimento cirúrgico.

Se tratando de uma patologia que tem como maior sintoma clínico a timidez dos portadores nas relações interpessoais, a busca de novos conhecimentos na área poderão promover a formulação de novas ações que promovam, de forma efetiva, a melhoria da qualidade de vida dos pacientes, no âmbito fisiológico e social.

\section{REFERÊNCIAS}

1. Reis GMD, Guerra ACS, Ferreira JPA. Estudo de pacientes com hiperidrose, tratados com toxina botulínica: análise retrospectiva de 10 anos. Rev. Bras. Cir. Plást. 2011; 26(4): 582-90.

2. Campos JR, Kauffman P, Werebe EC et al. Questionnaire of quality of life in patients with primary hyperhidrosis. J. Pneumologia, jul./ago 2003;29(4): 178-81.

3. Fenili R, Fistarol ED, Delorenze LM, Demarchi AR, Matiello $M$. Prevalência de hiperidrose em uma amostra populacional 
de Blumenau-SC, Brasil. An Bras. Dermatol. 2009; 84(4): 361-6.

4. Stori Jr. WS, Coelho MS, Guimarães PSF, Bergonse Neto N, Pizarrp LV. Bloqueio por clipagem de gânglios simpáticos torácicos no tratamento da hiperidrose. An. Bras. Dermatol 2006; 81 (5): 425-32. http://dx.doi.org/10.1590/S0365$\underline{05962006000500004}$

5. Hornberger J, Grimes K, Naumann M, Glaser DA, Lowe NJ, Naver $H$, et al. Recognition, diagnosis, and treatment of primary focal hyperhidrosis. J am Acad Dermatol. 2004; 51: 274-86. http://dx.doi.org/10.1016/j.jaad.2003.12.029

6. MOURA Jr, NB. Estudo anátomofuncional de glânglios da cadeia simpática torácica na hiperidrose primária. 2012. Tese (Doutorado em Cirurgia Torácica e Cardiovascular) - Faculdade de Medicina, Universidade de São Paulo, São Paulo, 2012. Disponível em: <http://www.teses.usp.br/teses/disponiveis/ 5/5156/tde-25052012-174823/>. Acesso em: 2014-07-14.

7. Montessi J, Almeida EP, Vieira JP, Abreu MM, Souza RLP, Montessi OVD. Simpatectomia torácica por videotoracoscopia para tratamento da hiperidrose primária: estudo retrospectivo de 521 casos comparando diferentes níveis de ablação. J Bras Pneumol. 2007;33(3):248-254. http://dx.doi.org/10.1590/S1806$\underline{37132007000300004}$

8. Chung $\mathrm{IH}$, Oh CS, Koh KS, Kim HJ, Paik $\mathrm{HC}$, Lee DY. Anatomic variations of the T2 nerve root (including the nerve of Kuntz) and their implications for sympathectomy. J Thorac Cardiovasc Surg. 2002;123(3):498501.

http://dx.doi.org/10.1067/mtc.2002.119340

9. Zacher J, Imhof M, Huber ER, Plas EG, Herbst $F$, Jaskesz $R$, et al. Video assistance reduces complication rate of thoracoscopic sympathicotomy for hyperhidrosis. Ann Thorac Surg. 1999;68(4):1177-81. http://dx.doi.org/10.1016/S00034975(99)00718-3

10. Lyra, RM et al . Diretrizes para a prevenção, diagnóstico e tratamento da hiperidrose compensatória. J. bras. Pneumol., São Paulo,v. 34, n. 11, Nov. 2008.

11. Crossetti, MGO. Revisão integrativa de pesquisa na enfermagem o rigor cientifico que lhe é exigido [editorial]. Rev Gaúcha Enferm., Porto Alegre (RS) 2012 jun;33(2):89.

12. Souza, MT et al. Revisão integrativa: o que é e como fazer. Einstein. 2010; 8(1 Pt 1): 102-6.

13. Ursi ES, Galvão CM. Prevenção de lesões de pele no perioperatório: revisão integrativa da literatura. Rev Latino-am Enfermagem 2006 janeiro-fevereiro; 14(1):124-

31. http://dx.doi.org/10.1590/s0104$\underline{11692006000100017}$

14. Yazbek G. Comparação dos resultados obtidos no tratamento da hiperidrose palmar pela simpatectomia torárica videotoracoscópica nos níveis de desnervação: T2 e T3. [tese]. São Paulo: Faculdade de Medicina, Universidade de São Paulo; 2009. 79p.

15. Boscardim PCF et al. Simpatectomia torácica ao nível de 4a e 5a costelas para o tratamento de hiperidrose axilar. J. Bras. Pneumol., São Paulo, v.37, n.1, Feb. 2011.

16. Araújo, CAA et al. Hiperhidrose compensatória após simpatectomia toracoscópica: características, prevalência e influência na satisfação do paciente. J. bras. Pneumol., São Paulo, v. 35,n. 3, Mar. 2009. 
17. Kestering DM et al. Videosimpatectomia torácia para tratamento da hiperidrose primária. Arquivos Catarinenses de Medicina Vol. 38, $\mathrm{n}^{\circ} .3$, de 2009.

18. Montessi, J et al . Simpatectomia torácica por videotoracoscopia para tratamento da hiperidrose primária: estudo retrospectivo de 521 casos comparando diferentes níveis de ablação. J. bras. Pneumol., São Paulo, v. 33,n. 3, June 2007.

19. Cardoso PO, et al. Avaliação de pacientes submetidos a tratamento cirúrgico de hiperidrose palmar quanto à qualidade de vida e ao surgimento e hiperidrose compensatória. Rev. Col. Bras. Cir. 2009; 36(1): 014-018.

20. Fiorelli RKA, et al. Avaliação do Impacto na Qualidade de Vida de Pacientes Portadores de Hiperidrose Primária Submetidos à Simpatectomia Videotoracoscópica. Meta: Avaliação, v. 3, n. 7, p. 1-24, jan./abr. 2011.

21. Lima AG. Estudo dos efeitos da sazonalidade sobre os resultados operatórios e grau de satisfação após simpatectomia videotoracoscópica e do desempenho dos métodos de expansão pulmonar empregados. [tese]. São Paulo: Faculdade de Medicina, Universidade de São Paulo; 2012. 153p.

22. Peres MFP, Brandão BFS, Kauffman $P$, Campos JRM, Jatene FB, Wolosker $\mathrm{N}$. Headaches and hyperhidrosis: clinical features and outcomes after surgery. Headache Medicine. 2010;1(1):5-8.

23. Loureiro MP, Roman N, Fontana SCW, Boscardim PCB. Simpactectomia lombar retroperitonioscópica para tratamento da hiperidrose plantar. Rev. Col. Bras. Cir. 2007;34(3).
Recebido para publicação em 10/08/2014 Revisado em 18/08/2014 Aceito em 19/08/2014 